\title{
Notes on the dynamics of pension funding *
}

\author{
Newton L. BOWERS, Jr. \\ Drake University, College of Business Administration, Des Moines, IA 50311, USA
}

James C. HICKMAN

University of Wisconsin, 1155 Observatory Drive, Madison, WI 53706, USA

\section{Cecil J. NESBITT}

University of Michigan, Department of Mathematics, Ann Arbor, MI 48109, USA

Received 12 March 1982

The authors follow up some previous work on the dynamics of pension funding by three notes. The first of these concerns contribution rates consisting of the normal cost plus a generalized amortization method for unfunded supplemental present value (actuarial accrued liability). The second note examines aggregate cost funding for active inembers when there exist consistent difference between the assumed and the actual rates of interest and of growth. The third nott: explores the operation of a variable annuity system in the context of our general model for pension funding dynamics.

Keywords: Pension funding dynamics, Contribution rates, Aggregate cost funding, Variable annuity.

\section{Introduction}

In previous papers $(1976,1979)$, the authors have discussed a model pension plan in respect to a covered population and payroll subject to growth. The theory developed in these papers is referred to as the dynamics of pension funding. In this paper we present some extensions and elaborations which follow directly from the theory already developed. We believe that these extensions will provide additional insights into some of the long term consequences of various decisions regarding pension lunding. The extensions will be organized in a series of notes.

The first note discusses a general contribution formula and a number of special cases and modifications. A second note concerns aggregate cost funding when the actuary uses assumptions about cost factors which differ consistently from cost experience. The final note concerns the variation of the annuity unit under a variable annuity program patterned after the plan employed by the College Retirement Equities Fund (CREF).

\section{A general contribution formula}

For the model plan discussed in Bowers, Hickman and Nesbitt (1976, 1979), we consider a contribution rate $(a C)(t)$ at time $t$ given by the formula

$$
(a \hat{C})(t)=P(t)+\lambda(a U)(t)
$$

\footnotetext{
- Published with kind permission of ARCH.
} 
This formula was introduced in Bowers, Hickman and Nesbitt (1979), Section IV, under the title 'Normal Cost Plus Amortization Over a Moving Term' with $\lambda-1 / \bar{a}_{n \mid}$ providing for amortization over $n$ years from the valuation time $t$. In formula (1) the prefix $a$ denotes that attention is fixed on active members. The symbol $P(t)$ denotes the annual rate of normal cost for the plan at time $t$ and $(a U)(t)=(a V)(t)-(a F)(t)$ denotes the unfunded supplemental present value at time $t$ for active members. Also, $(a V)(t)$ and $(a F)(t)$ denote the supplemental present value and the fund on hand at time $t$ for such members. Bold-face printing of symbols indicates that the; relate to the whole group of active members rather than to a unit of benefit.

It would be natural to generalize formula (1) by replacing the constant $\lambda$ by a function $\lambda(t)$. This generalization will be used later in this note but we shall start with the simpler case where $\lambda$ is a constant. As is pointed out in Bowers, Hickman and Nesbitt (1979), Section IV, the contribution rate defined by formula (1) is related to the generalized aggregate cost method first described by Trowbridge [10].

From formula (42) in Bowers, Hickman and Nesbitt (1979)

$$
\frac{\mathrm{d}}{\mathrm{d} t}(a F)(t)=(a C)(t)+\delta(a F)(t)-{ }^{\mathrm{T}} \boldsymbol{P}(t),
$$

and from formula (7) in the same paper

$$
\frac{\mathrm{d}}{\mathrm{d} t}(a V)(t)=P(t)+\delta(a V)(t)-{ }^{\top} \boldsymbol{P}(t)
$$

In formulas (2) and (3), ${ }^{\mathrm{T}} \boldsymbol{P}(t)$ is the terminal funding normal cost rate at time $t$. Using formula (1) and subtracting formula (2) from formula (3), we obtain the following differential equation for the unfunded supplemental present value:

$$
\frac{\mathrm{d}}{\mathrm{d} t}(a U)(t)=(\delta-\lambda)(a U)(t)
$$

Solving differential equation (4) yields

$$
(a U)(t)=(a U)(0) \mathrm{e}^{(\delta-\lambda) t}
$$

(cf. formulas (49) and (50) of Bowers, Hickman and Nesbitt (1979)). Using formula (5) and the definition of unfunded supplemental present value, we obtain

$$
(a F)(t)=(a V)(t)-(a U)(0) \mathrm{e}^{(\delta-\lambda) t} .
$$

To derive some measure of the economic burden of the unfunded supplemental present value, we consider the ratio of such present value to the payroll rate at time $t$, and obtain

$$
\frac{(a U)(t)}{W(t)}=\frac{(a U)(0)}{W(0)} \mathrm{e}^{(\delta-\tau-\lambda) t}
$$

where $W(t)=W(0) \mathrm{e}^{7 t}$ (formula (79) of Bowers, Hickman and Nesbitt (1976)) is the annual rate of payroll at time $t$, and $\tau$ is the total rate of growth of payroll. In formula (7), we have assumed, for the first time in this paper, the exponential growth case. With this assumption, formula (6) can be rewritten as

$$
(a F)(t)=(a V)(0)\left[\mathrm{e}^{t t}-\mathrm{e}^{(\delta-\lambda) \prime}\right]+(a F)(0) \mathrm{e}^{(\delta-\lambda) t},
$$

as follows from formula (18) in our 1979 paper. Unless it is indicated otherwise, the exponential growth cara will be assumed in the following.

a he total growth rate $\tau$ of payroll in the exponential growth case equals $\alpha+\gamma$ where $\alpha$ measures the rate of covered population growth and $\gamma$ is the growth rate of pay levels. In the language of Allison and Winklevoss (1975), the rate $\gamma$ is to capture the impact of inflation and population-wide changes in productivity on the payroll rate.

Three special cases of formula (1) with some significance for practice will be considered.

Case $A$. No payment on unfunded supplemental present value $(\lambda=0)$. 
If $\lambda=0$, then $(a C)(t)=P(t)$, and by substituting in formulas (5), (6) and (7), we obtain

$$
\begin{aligned}
& (a U)(t)=(a U)(0) \mathrm{e}^{\delta t}, \\
& (a F)(t)=(a V)(0) \mathrm{e}^{\tau t}-(a U)(0) \mathrm{e}^{\delta t}, \\
& \frac{(a U)(t)}{W(t)}=\frac{(a U)(0)}{W(0)} \mathrm{e}^{\theta t},
\end{aligned}
$$

where $\theta=\delta-\tau$. In formula (9), there is a growth competition between the supplemental present value growing at rate $\tau$ and $(a U)(0)$ accumulating at force of interest $\delta$. If $(a F)(0)=0$, we have

$$
(a F)(t)=(a V)(0)\left[\mathrm{e}^{\tau t}-\mathrm{e}^{\delta t}\right]
$$

which illustrates the importance of the relationship between $\tau$ and $\delta$. Unless $\tau>\delta$, a deficit will arise immediately in this case. Even if $(a F)(0)>0$, a deficit will occur ultimately if the growth rate is less than the interest rate.

If $0<\delta<\tau$, a related observation can be made about formula (10). In this situation $\theta<0$, and the ratio $(a U)(t) / W(t)$ will decrease even though there is no payment on the unfunded supplemental present value.

Case B. Payment on unfunded supplemental present value at rate $\theta=\delta-\tau(\lambda=\theta)$.

Here $(a C)(t)=P(t)+\theta(a U)(t)$, and formulas (5), (6a) and (7) become

$$
\begin{aligned}
& (a U)(t)=(a U)(0) \mathrm{e}^{\tau t} \\
& (a F)(t)=(a F)(0) \mathrm{e}^{\tau t} \\
& \frac{(a U)(t)}{W(t)}=\frac{(a U)(0)}{W(0)}
\end{aligned}
$$

In this case, the fund $(a F)(t)$ grows at rate $\tau$, the same as the other functions such as $P(t)$ and $(a V)(t)$ (see formula (18) in Bowers, Hickman and Nesbitt (1979)). Of course, if $(a F)(0)=0$, the fund will remain at zero. The ratio $(a U)(t) / W(t)$ remains fixed. If $\tau>\delta$, then $(a C)(t)<P(t)$, that is, the contribution rate is less than the normal cost rate.

These observations indicate that if $\lambda>\theta$, then $(a F)(t)$ increases more rapidly than $(a V)(t)$ which grows at rate $\tau$. This statement can be verified by reference to formula (6) with $\delta-\lambda<\delta-\theta=\tau$ to see that

$$
\mathrm{d}(\boldsymbol{a} F)(t) / \mathrm{d} t>\tau(a F)(t) \text {. }
$$

Case $C$. Payment of interest on unfunded supplemental present value $(\lambda=\delta)$.

If $\lambda=\delta$, then $(a C)(t)=P(t)+\delta(a U)(t)$ and formulas (5), (6), (6a) and (7) become

$$
\begin{aligned}
& (a U)(t)=(a U)(0), \\
& (a F)(t)=(a V)(t)-(a U)(0)=(a V)(0)\left[\mathrm{e}^{t t}-1\right]+(a F)(0), \\
& \frac{(a U)(t)}{W(t)}=\frac{(a U)(0)}{W(0)} \mathrm{e}^{-\tau t} .
\end{aligned}
$$

From formula (15), we see that the growth in the fund keeps up with the growth in the supplemental present value, and from formula (16) that the economic burden of unfunded supplemental present value declines.

We summarize our observations concerning these special contribution cases in Table 1 . The contribution rates involved are clearly minimal but their long term implications for the model plan under various circumstances may be illuminating.

By increasing or decreasing $\lambda$, one increases or decreases the rate at which the unfunded supplemental present value approaches zero (see formula (5)). Trowbridge (1963) has discussed this point extensively.

We turn now to a contribution pattern called "Normal Cost Plus Amortization Over a Fixed Term" in 
Table 1

Summary of special cases

\begin{tabular}{|c|c|c|c|c|c|c|}
\hline & \multicolumn{2}{|c|}{ Case $A, \lambda=0$} & \multicolumn{2}{|c|}{ Case $B, \lambda=\delta-\tau$} & \multicolumn{2}{|c|}{ Case $C, \lambda=\delta$} \\
\hline & $0<\delta<\tau$ & $0<\tau<\delta$ & $0<\delta<\tau$ & $0<\tau<\delta$ & $0<\delta<\tau$ & $0<\tau<\delta$ \\
\hline $\begin{array}{l}(a U)(t) \\
(a F)(t)\end{array}$ & $\begin{array}{l}\text { Increasing } \\
\text { Increasing a }\end{array}$ & $\begin{array}{l}\text { Increasing } \\
\text { Uitimately } \\
\text { negative and } \\
\text { decreasing }\end{array}$ & $\begin{array}{l}\text { Increasing } \\
\text { Constant at } \\
\text { zero or } \\
\text { increasing b }\end{array}$ & $\begin{array}{l}\text { Increasing } \\
\text { Constant at } \\
\text { zero or } \\
\text { increasing }\end{array}$ & $\begin{array}{l}\text { Constant } \\
\text { Increasing }\end{array}$ & $\begin{array}{l}\text { Constant } \\
\text { Increasing }\end{array}$ \\
\hline$\frac{(a U)(t)}{W(t)}$ & Decreasing & Increasing & Constant & Constant & Decreasing & Decreasing \\
\hline
\end{tabular}

a This fact is related to the proposition developed by Samuelson, Aaron and others, and renewed by Hickman (1968), that if $\delta<\tau$, a pay-as-you-go social insurance plan increases the welfare of persons who receive average real wages.

b In this case $(a C)(t)<P(t)$, yet because of the relatively high growth rate, an increasing fund develops (provided the initial fund is positive).

Section IV of our 1979 paper. This corresponds to setting $\lambda(t)=1 / \bar{a}_{n-1 \mid}$ in formula (1), (where compound interest functions are based on force $\theta$ ). We have then

$$
(a C)(t)=P(t)+(a U)(t) / \bar{a}_{n-t \mid} .
$$

However, we suppose that the plan's sponsor follows the consistent policy of contributing at the rate

$$
(a \tilde{C})(t)=\boldsymbol{P}(t)+f(a \tilde{U})(t) / \bar{a} \overline{n-1 \mid}, \quad 0<f<1 .
$$

Following the same steps that led to formula (5), we obtain for our measure of the economic burden of unfunded supplemental present value,

$$
\frac{(a \tilde{U})(t)}{W(t)}=\frac{(a U)(0)}{W(0)} \exp \left[\theta t-f \int_{0}^{t} \bar{a}_{n-h \mid}^{-1} \mathrm{~d} h\right] .
$$

It can be shown that

$$
\int_{0}^{t} \bar{a}_{n-h \mid}^{-1} \mathrm{~d} h=\log \left[\bar{s}_{\bar{n} \mid} / \bar{s}_{\overline{n-1}}\right]
$$

and it follows that

$$
\frac{(a \tilde{U})(t)}{W(t)}=\frac{(a U)(0)}{W(0)} \mathrm{e}^{\theta t}\left[\overline{s_{n-t \mid}} / \bar{s}_{\bar{n} \mid}\right]^{f}
$$

In the situation defined by formula (1), a reduction in $\lambda$, based on a decision of the plan sponsor, has the effect of slowing the decay of $(a U)(t) / W(t)$, as can be seen from formula (7). In the case defined by formula (18), the effect of changing the funding policy by selecting $f, 0<f<1$, is more complex. The amortization of unfunded supplemental present value is still completed in $n$ years, but there is a transfer from the earlier to the latter part of the term. This is illustrated by the graphs in Figure 1.

If $f=1$, formula (21) becomes

$$
\frac{(a U)(t)}{W(t)}=\frac{(a U)(0)}{W(0)}\left(\bar{a}_{\overline{n-t \mid}} / \bar{a}_{\bar{n} \mid}\right)
$$

which is equivalent to formula (44) of our 1979 paper.

In yet another modification of formula (17), let us suppose that the plan sponsor adopts the policy of contributing a fixed amount $k$ less per year than is specified by formula (17), that is

$$
(a \tilde{C})(t)=P(t)+[(a \tilde{U})(t) / \bar{a} \overline{n-t}]-k
$$




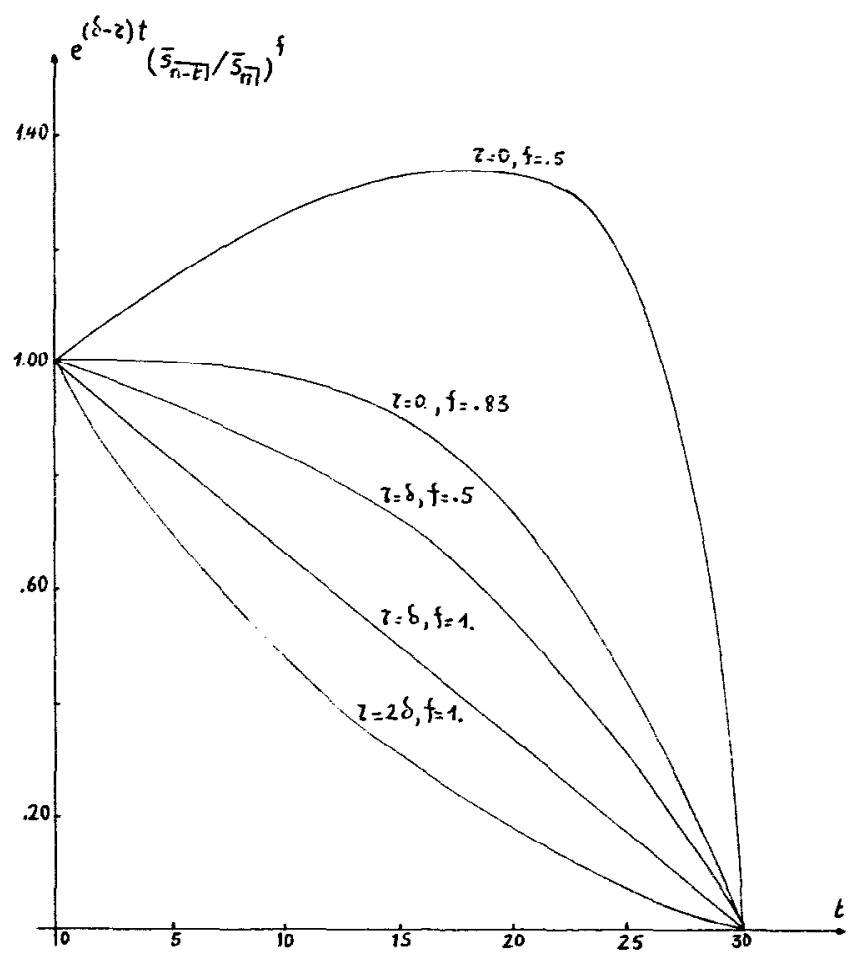
Fig. 1. Path of ratio of unfunded supplemental present value to payroll based on formula $(21)$ with $(a U)(0) / W(0)=1.0, \delta=0.06$,
$n=30$.

Formula (4) becomes

$$
\frac{\mathrm{d}}{\mathrm{d} t}(a \tilde{U})(t)=\left(\delta-\bar{a}_{n-t \mid}^{-1}\right)(a \tilde{U})(t)+k \text {. }
$$

The solution of this differential equation for $(a \tilde{U})(t)$, and the calculation of the measure of economic burden of unfunded supplemental present value, $(a \tilde{U})(t) / W(t)$, shows this ratio to be formula (22) plus the amount

$$
k \bar{a} \overline{n-1 \mid}\left(\log \left[\bar{s}_{n \mid} \frac{-1}{s_{n-1}}\right]\right) / W(0) \mathrm{e}^{\tau \prime} .
$$

As $t \rightarrow n$, this increment goes to zero. For $\tau=\delta$, formula (25) is

$$
k(n-t)(\log [n /(n-t)]) / W(0) \mathrm{e}^{\tau t} .
$$

\section{Aggregate cost funding with assumed rates different from experience rates}

In this section we consider what develops under aggregate cost funding if experience interest and growth rates differ from valuation assumptions in a consistent fashion. Interest at force $\delta$ is assumed for the future, but investment income at force $\delta^{\prime}$ has been experienced in the past and will be experienced in the future. Payroll is assumed to grow at a continuous annual rate $\tau$, but in fact in the past it has grown at rate $\tau^{\prime}$ and will do so in the future. The development will be restricted to functions for active members. This is in accord with the practice, which we expect will become increasingly common, to consider a separate fund for the retirees with experience gains in the separate fund distributed to the retirees. Some of the issues involved in distributing experience gains to retired lives will be considered in Section 4. For simplicity, we will consider only the exponential growth case here. 
Under these assumptions, the annual rate of normal cost can be expressed as (see formula (4) in Bowers, Hickman and Nesbitt (1979))

$$
\boldsymbol{P}(t)=\int_{a}^{r} \mathrm{e}^{\tau^{\prime} t} \mathrm{e}^{-(\delta-\tau)(r-x) \mathrm{T}} \boldsymbol{P} m(x) \mathrm{d} x
$$

where ${ }^{\top} P=l_{r} s(r) b \bar{a}_{r}, \bar{a}_{r}$ is valued at force of interest $\delta$, and $m(x)$ is the pension purchase density function. Note that the term $\mathrm{e}^{\tau^{\prime} t}$ takes into account actual growth in payroll to time $t$ at rate $\tau^{\prime}$, and that growth in the future is assessed at rate $\tau$ and interest is assessed at $\delta$ (according to the valuation assumptions). With $M(x)=\int_{a}^{x} m(y) \mathrm{d} y$, integration by parts on formula (27) yields

$$
\begin{aligned}
\boldsymbol{P}(t) & =\int_{a}^{r} \mathrm{e}^{\tau^{\prime} t} \mathrm{e}^{-(\delta-\tau)(r-x) \mathrm{T} P \mathrm{~d} M(x)} \\
& =\left.\mathrm{e}^{\tau^{\prime} t} \mathrm{e}^{-(\delta-\tau)(r-x) \mathrm{T} P} M(x)\right|_{a} ^{r}-\int_{a}^{r} \mathrm{e}^{\tau^{\prime} t} \mathrm{e}^{-(\delta-\tau)(r-x)}(\delta-\tau)^{\mathrm{T}} \boldsymbol{P} M(x) \mathrm{d} x \\
& =\mathrm{e}^{\tau^{\prime} t \mathrm{~T} P}-(\delta-\tau)(\boldsymbol{a} \boldsymbol{V})(t) .
\end{aligned}
$$

The integral on the right hand side of the second equality of formula (28) is an extension of formula (5) in our 1979 paper. That formula states the supplemental value at time $t$ is given by

$$
(a V)(t)=\int_{a}^{r} \mathrm{e}^{-\delta(r-x) \mathrm{T}} \boldsymbol{P}(t+r-x) M(x) \mathrm{d} x .
$$

In the present case ${ }^{\mathrm{T} P} \mathrm{e}^{\tau^{\prime} t+\tau(r-x)}$ is playing the role of ${ }^{\mathrm{T}} \boldsymbol{P}(t+r-x)$, a distinction being made about experience growth in the past at rate $\tau^{\prime}$ and assumed growth in the future at rate $\tau$. Formula (28) may be rearranged to yield

$$
\boldsymbol{P}(t)+\delta(\boldsymbol{a} V)(t)={ }^{\mathrm{T}} \boldsymbol{P}(t)+\tau(\boldsymbol{a} V)(t)
$$

which appears on the surface to be identical to the income allocation equation, formula (7) in our 1979 paper. However, it is important to note, using formula (28), that

$$
\frac{\mathrm{d}}{\mathrm{d} t}(a V)(t) \neq \tau(a V)(t)
$$

In fact, because the functions $P(t),(a V)(t)$, and ${ }^{\mathrm{T}} \boldsymbol{P}(t)$ all have the factor $\mathrm{e}^{\tau^{\prime} t}$, the functions grow at rate $\tau^{\prime}$ rather than rate $\tau$.

Exploiting this remark and formula (29), we find

$$
\begin{aligned}
\frac{\mathrm{d}}{\mathrm{d} t}(a V)(t) & =\tau^{\prime}(a V)(t)=\tau(a V)(t)+\left(\tau^{\prime}-\tau\right)(a V)(t) \\
& =\left[P(t)+\delta^{\prime}(a V)(t)-{ }^{\top} P(t)\right]+\left[\left(\tau^{\prime}-\tau\right)-\left(\delta^{\prime}-\delta\right)\right](a V)(t) \\
& =P(t)+\delta^{\prime}(a V)(t)-{ }^{\top} P(t)+\left(\theta-\theta^{\prime}\right)(a V)(t) .
\end{aligned}
$$

We assume that aggregate cost funding is according to the formula

$$
(a C)(t)=\boldsymbol{P}(t)+\frac{(a U)(t)}{\tilde{a}}
$$

(see formula (59) in our 1979 paper). In this expression, the mean temporary annuity $\tilde{a}=(\boldsymbol{P a})(t) / \boldsymbol{P}(t)$ is independent of $t$ in the exponential growth case (see formula (84) in our 1976 paper) and is based on the assumed rates $\delta$ and $\tau$. Then the progress of the active life fund is governed by the differential equation

$$
\frac{\mathrm{d}(a F)(t)}{\mathrm{d} t}=(a C)(t)+\delta^{\prime}(a F)(t)-{ }^{\mathrm{T}} \boldsymbol{P}(t)=\boldsymbol{P}(t)+\frac{(a U)(t)}{\tilde{a}}+\delta^{\prime}(a F)(t)-\mathrm{T} P(t) .
$$

Note that in formula (32) the fund is earning interest at the experience rate $\delta^{\prime}$. However the life annuity factor $\bar{a}_{r}$ in ${ }^{\mathrm{T}} \boldsymbol{P}(t)=\mathrm{e}^{\tau^{\prime} t} l_{r} s(r) b \bar{a}_{r}$ is valued at the assumed force of interest $\delta$. This means that if $\delta^{\prime}>\delta$, the fund for retirees will experience consistent gains by reason of the force of interest differential $\delta^{\prime}-\delta$. These gains could be distributed to the retirees. In the first two papers in this series, it was stressed that the 
annuity to retired lives could contain an adjustment factor $\beta(x)$ providing for continuous and deterministic adjustment of pension amounts. In the present case the adjustment would be based on interest gains on the retired life fund. Aspects of this possibility are discussed in Section 4.

Subtracting formula (32) from formula (30), we obtain

$$
\frac{\mathrm{d}}{\mathrm{d} t}(\boldsymbol{a} \bar{U})(t)=\left(\tilde{a}^{-1}-\delta^{\prime}\right)(a U)(t)=k(a V)(t)=k(a V)(0) \mathrm{e}^{r^{\prime} t},
$$

where $k=\theta-\theta^{\prime}=(\delta-\tau)-\left(\delta^{\prime}-\tau^{\prime}\right)=\left(\delta-\delta^{\prime}\right)-\left(\tau-\tau^{\prime}\right)$.

Solving the differential equation in formula (33), replacing $(a V)(0)$ by $(a V)(t) \mathrm{e}^{-\tau^{\prime} t}$, and assuming $(a F)(0)=0$, yields

$$
(a F)(t)=(a V)(t)\left[\frac{\tilde{a}^{-1}-\theta}{\tilde{a}^{-1}-\theta^{\prime}}\right]\left[1-\mathrm{e}^{-\left(\tilde{u}^{-1}-\theta^{\prime}\right) t}\right] .
$$

Thus if $\tilde{a}^{-1}>\theta^{\prime}=\left(\delta^{\prime}-\tau^{\prime}\right)$,

$$
\lim _{t \rightarrow \infty} \frac{(a F)(t)}{(a V)(t)}=\frac{\tilde{a}^{-1}-\theta}{\tilde{a}^{-1}-\theta^{\prime}}
$$

If $\theta^{\prime}=\delta^{\prime}-\tau^{\prime}=\delta-\tau=\theta$, the limit in formula (35) is ore, and the active life fund approaches the supplemental present value for actives. If $\tilde{a}^{-1}>\theta^{\prime}=\left(\delta^{\prime}-\tau^{\prime}\right)>\theta=\delta-\tau$, the experience difference between growth and interest rates is greater than the corresponding assumed difference, and the fund will become larger than the supplemental present value. If $a^{-1}>\theta^{\prime}=\left(\delta^{\prime}-\tau^{\prime}\right)<\theta=\delta-\tau$, the experience difference between growth and interest rates is less than the corresponding assumed difference, and the fund for active lives will remain smaller than the supplemental present value. In this simplified exponential growth model, note that it is the relationship between the difference of spread between the interest and growth rates in the valuation assumptions and the experience that determines the asymptotic relationship between the size of the fund and the size of the supplemental present value.

\section{Annuity unit valuation in a CREF-like plan}

In Section 3 we considered some implications of a consistent difference between assumed and experience interest and growth rates. In this section a related question will be considered. Can the dynamics of pension funding be used to illustrate the operation of a variable annuity system such as CREF? The CREF system is described by Duncan (1952). By restricting attention to retired lives and by making appropriate modifications, it does prove possible to adapt our theory for the illustrative purpose indicated. The discussion by Cody (1969) on the fundamentals of variable annuities also uses a continuous model that has many similarities to ours.

We consider a variable annuity accumulation fund from which annuity units are purchased at age $r$ on the basis of assumed interest at force $\delta$ and assumed mortality at force $\mu_{x}, x \geqslant r$. The actual force of investment yield will be noted by $\delta_{t}^{\prime}$, assumed time dependent, and the actual mortality force by $\mu_{x}^{\prime}, x \geqslant r$. This latter force may also be time dependent to some degree but for our illustrative purposes we shall assume that such time dependent force can be replaced by an average force for the time period studied. The rate of payment at time $t$ for an annuity unit will depend on the experience forces of investment return and of mortality, and will be denoted by $b(t)$. We shall call $b(t)$ the annuity unit value at time $t$.

The present value at time $t$ for the annuities then in force will be denoted by $(r V)(t)$.

$$
(\boldsymbol{r})(t)=b(t) H(t)
$$

where

$$
H(t)=\int_{r}^{\infty} N(t+r-x)\left(l_{x}^{\prime} / l_{r}^{\prime}\right) \bar{a}_{x} \mathrm{~d} x
$$

In formula (37), $N(t+r-x)$ denotes the number of annuity units which were purchased by members 
reaching age $r$ at time $t-(x-r)$. The factor $\left(l_{x}^{\prime} / l_{r}^{\prime}\right)$ measures actual survival from age $r$ to age $x$ by such purchasers, and $\bar{a}_{x}$ denotes the unit life annuity value on the basis of the assumed forces $\delta$ and $\mu_{x}$. The symbol $\boldsymbol{H}(t)$ may be interpreted as representing the present value, expressed in annuity units, of the annuities in force at time $t$. Then $(r V)(t)$ is the present value, in dollars, of the annuities in force at time $t$ taking into account the current annuity unit value $b(t)$. It will be assumed that $(r V)(t)=(r F)(t)$, the fund for retired lives, and therefore

$$
b(t)=\frac{(r F)(t)}{H(t)} .
$$

Unlike the formula presented by Duncan (1952), no allowance is made for expenses, and the annuity unit value varies continuously rather than annually.

For our case, the annual rate $B(t)$ of annuity payments at time $t$ will be given by

$$
\boldsymbol{B}(t)=\boldsymbol{b}(t) \boldsymbol{I}(t)
$$

where

$$
I(t)=\int_{r}^{\infty} N(t+r-x)\left(l_{x}^{\prime} / l_{r}^{\prime}\right) \mathrm{d} x
$$

is the annual rate of annuity payments in terms of annuity units.

The progress of $(\boldsymbol{r} V)(t)$, the present value in dollars of annuities in force at time $t$, and also of $(\boldsymbol{r} F)(t)$, will be governed by the differential equation

$$
\frac{\mathrm{d}}{\mathrm{d} t}(\boldsymbol{r} V)(t)=N(t) b(t) \vec{a}_{r}+\delta_{t}^{\prime}(\boldsymbol{r} V)(t)-B(t) .
$$

Equation (41) has been written down from general principles and will shortly be compared with what would be obtained by differentiating the right member of formula (36). Through this comparison, we shall obtain a differential equation for $b(t)$. Equation (41) expresses the same idea as presented by Kischuk in his discussion (1976, p. 206). The new initial annuity income purchased at time $t$ is $N(t) b(t)=C(t) / \bar{a}_{r}$, where $C(t)$ is the amount of money transferred from an accumulation to an annuity account at ime $t$. The new annuity income, $C(t) / \bar{a}_{r}$, depends on the current value of the accumulations of the lives entering the retired state, and on the interest and mortality basis of $\bar{a}_{r}$, but it does not depend on $b(t)$.

Differentiating the expression in formula (36), we obtain

$$
\frac{\mathrm{d}}{\mathrm{d} t}(\boldsymbol{r} V)(t)=\boldsymbol{H}(t) \frac{\mathrm{d} \boldsymbol{b}(t)}{\mathrm{d} t}+\boldsymbol{b}(t) \frac{\mathrm{d} \boldsymbol{H}(t)}{\mathrm{d} t} \text {. }
$$

But, from formula (37), we have

$$
\begin{aligned}
\frac{\mathrm{d}}{\mathrm{d} t} \boldsymbol{H}(t) & =\int_{r}^{\infty}\left[\frac{\partial}{\partial t} N(t+r-x)\right] \frac{l_{x}^{\prime}}{l_{r}^{\prime}} \bar{a}_{x} \mathrm{~d} x=\int_{r}^{\infty}\left[-\frac{\partial}{\partial x} N(t+r-x)\right] \frac{l_{x}^{\prime}}{l_{r}^{\prime}} \bar{a}_{x} \mathrm{~d} x \\
& =-\int_{r}^{\infty} \frac{l_{x}^{\prime}}{l_{r}^{\prime}} \bar{a}_{x} \mathrm{~d} N(t+r-x) \quad \text { (see p. } 187 \text { of Bowers, Hickman and Nesbitt (1976)) } \\
& =-\left.\frac{l_{x}^{\prime}}{l_{r}^{\prime}} \bar{a}_{x} N(t+r-x)\right|_{r} ^{\infty}+\int_{r}^{\infty}\left[N(t+r-x) / l_{r}^{\prime}\right]\left[-l_{x}^{\prime} \mu_{x}^{\prime} \bar{a}_{x}+l_{x}^{\prime}\left\{\bar{a}_{x}\left(\mu_{x}+\delta\right)-1\right\}\right] \mathrm{d} x \\
& =N(t) \bar{a}_{r}+\delta H(t)-I(t)+M(t)
\end{aligned}
$$

where

$$
M(t)=\int_{r}^{\infty} N(t+r-x)\left(l_{x}^{\prime} / l_{r}^{\prime}\right) \bar{a}_{x}\left(\mu_{x}-\mu_{x}^{\prime}\right) \mathrm{d} x .
$$

Then

$$
\frac{\mathrm{d}(\boldsymbol{r} V)(t)}{\mathrm{d} t}=\boldsymbol{H}(t) \frac{\mathrm{d} \boldsymbol{b}(t)}{\mathrm{d} t}+N(t) \boldsymbol{b}(t) \tilde{a}_{r}+\delta(\boldsymbol{r} V)(t)-\boldsymbol{B}(t)+\boldsymbol{b}(t) \boldsymbol{M}(t) .
$$


Equating the right hand members of formulas (41) and (43) yields

$$
\frac{\mathrm{d} b(t)}{\mathrm{d} t}=\left[\delta_{t}^{\prime}-\delta-(M(t) / \boldsymbol{H}(t))\right] b(t)
$$

The differential equation (44) for $b(t)$ can be solved in the form

$$
\boldsymbol{b}\left(t_{1}\right)=\boldsymbol{b}\left(t_{0}\right) \exp \left[\int_{t_{0}}^{t_{1}}\left[\delta_{t}^{\prime}-\delta-(M(t) / \boldsymbol{H}(t)] \mathrm{d} t\right]\right. \text {. }
$$

But

$$
M(t) / H(t)=\int_{r}^{\infty} N(t+r-x)\left(l_{x}^{\prime} / l_{r}^{\prime}\right)\left(\mu_{x}-\mu_{x}^{\prime}\right) \bar{a}_{x} \mathrm{~d} x / \int_{r}^{\infty} N(t+r-x)\left(l_{x}^{\prime} / l_{r}^{\prime}\right) \bar{a}_{x} \mathrm{~d} x
$$

(which relates mortality gain or loss to $\boldsymbol{H}(t)$ ) is a weighted average at time $t$ of $\mu_{x}-\mu_{x}^{\prime}, r \leqslant x \leqslant w$, say

$$
\boldsymbol{M}(t) / \boldsymbol{H}(t)=\bar{\mu}_{t}-\bar{\mu}_{t}^{\prime} .
$$

Then equation (45) may be rewritten as

$$
b\left(t_{1}\right)=b\left(t_{0}\right) \exp \left[\int_{t_{0}}^{t_{1}}\left[\delta_{t}^{\prime}-\delta+\bar{\mu}_{t}^{\prime}-\bar{\mu}_{t}\right] \mathrm{d} t\right]
$$

which indicates how the annuity unit value grows as a result of investment gain or loss and mortality gain or loss.

In CREF the annuity unit is revalued once each year in April to reflect CREF's total experience for the preceding fiscal year April 1 to March 31 (see the TIAA Report (1981)). The CREF annuity unit values since 1952 are shown in Table 2. In the 1981 TIAA Report it is stated that changes in the annuity unit value result primarily from the investment experience of CREF's common stocks, and CREF's mortality experience and expenses produce smaller effects. The assumed effective annual rate of interest is 4 percent, and the current mortality assumptions are the 1971 Individual Annuity Mortality Table with ages set back 0 years for males and $1 \frac{1}{2}$ years for females.

From Table 2 it can be observed that the highest annuity unit value $\$ 35.86$ was in 1981 (with a close second of $\$ 35.74$ in 1972), and that in the six years 1961, 1962, 1964, 1974, 1976 and 1980 the annuity unit stood at the $\$ 26$ level.

In some variable annuity plans (see Campbell (1969) and Cody (1969)) the annuitants do not participate in the gains and losses in mortality experience, as is the case in the CREF plan. When this is the case, the term $\boldsymbol{M}(t) / \boldsymbol{H}(t)$ would not appear in formula (44), and formula (45) would become

$$
\boldsymbol{b}\left(t_{1}\right)=\boldsymbol{b}\left(t_{0}\right) \exp \left[\int_{t_{0}}^{t_{1}}\left(\delta_{r}^{\prime}-\delta\right) \mathrm{d} t\right] \text {. }
$$

Table 2

CREF annuity unit values since 1952 (annuity year: May through April)

\begin{tabular}{rrrrrr}
\hline 1952 & $\$ 10.00$ & 1962 & $\$ 26.13$ & 1972 & $\$ 35.74$ \\
1953 & 9.46 & 1963 & 22.68 & 1973 & 31.58 \\
1954 & 10.74 & 1964 & 26.48 & 1974 & 26.21 \\
1955 & 14.11 & 1965 & 28.21 & 1975 & 21.84 \\
1956 & 18.51 & 1966 & 30.43 & 1976 & 26.24 \\
1957 & 16.88 & 1967 & 31.92 & 1977 & 24.80 \\
1958 & 16.71 & 1968 & 29.90 & 1978 & 23.28 \\
1959 & 22.03 & 1969 & 32.50 & 1979 & 27.28 \\
1960 & 22.18 & 1970 & 28.91 & 1980 & 26.27 \\
1961 & 26.25 & 1971 & 30.64 & 1981 & 35.86 \\
& & & & 1982 & 30.56
\end{tabular}


that is, the annuity unit value would vary by reason of the difference between the experienced investment return and the assumed return.

\section{Summary}

Section 2 has dealt with contribution rates for active members consisting of the normal cost plus a generalized amortization method for unfunded supplemental value. Several significant special cases involving prescribed relationships among amortization, interest and growth rates were examined. Section 3 discussed aggregate cost funding for active members when there exist consistent differences between the assumed and the actual interest and growth rates. Section 4 reviewed the operation of a variable annuity system by means of the general model for pension dynamics. It appears that many other pension funding questions can be studied by means of this model.

\section{References}

Allison, G.D. and Winklevoss, H.E. (1975). The interrelationships among inflation rates, salary rates, interest rates and pension costs. Trans. Soc. Actuaries 27, 197-210.

Bowers, N.L., Jr., Hickman, J.C. and Nesbitt, C.J. (1976). Introduction to the dynamics of pension funding. Trans. Soc. Actuaries 28, $177-203$.

Bowers, N.L., Jr., Hickman, J.C. and Nesbitt, C.J. (1979). The dynamics oî pension funding: Contribution theory. Trans. SOc. Actuaries 31, 93-119.

Campbell, P. (1969). The Variable Annuity, Its Development, Its Environment, and Its Future. Connecticut General Life Insurance Co., Hartford, CT.

Cody, D.D. (1969). Discussion of "Actuarial aspects of the insured variable annuity" by John Macarchuk. Trans. Soc. Actuaries 21, $541-545$.

Duncan, R.M. (1952), A retirement system granting unit annuities and investing in equities. Trans. Soc. Actuaries 4, $317-344$.

Hickman, J.C. (1968). Funding theories for social insurance. Proc. Casualty Actuarial Soc. 55, 303-311.

Kischuk, R.K. (1976). Discussion of "Introduction to the dynamics of pension funding" by Bowers, Hickmann and Nesbitt (1976). Trans. Soc. Actuaries 28, 205-211.

Teachers Insurance and Annuity Association - College Retirement Equities Fund, New York. 1981 Annual Report.

Trowbridge, C.L. (1963). The unfunded present value family of pension funding methods, Trans. Soc. Actuaries 15, $151-169$. 